\title{
Acetylcholinesterase inhibition and antimicrobial activity of hydroxyl amides synthesized from natural products derivatives
}

\author{
MARIA AMÉLIA D. BOAVENTURA, LAURA F.W. XAVIER, HENRIETE S. VIEIRA, JACQUELINE A. \\ TAKAHASHI, WILTON J.D. NASCIMENTO JÚNIOR, TAMIRES P. ARAUJO and AMANDA C.S. COELHO \\ Departamento de Química, Instituto de Ciências Exatas, Universidade Federal de Minas \\ Gerais, Av. Antônio Carlos, 6627, 31270-901 Belo Horizonte, MG, Brazil
}

Manuscript received on June 28, 2017; accepted for publication on November 7, 2017

\begin{abstract}
Thirteen natural products derivatives of hydroxyl amide class, three described for the first time, were synthesized by reaction of three indole acids and 3,4,5-trimethoxybenzoic acid with six different amino alcohols in the presence of triphenylphosphine and $N$-bromosuccinimide. The derivatives were tested against the Gram (+) bacteria Staphylococcus aureus and Bacillus cereus, Gram (-) Pseudomonas aeruginosa and Escherichia coli, besides the yeast Candida albicans. One of the compounds (7) was selectively active against $C$. albicans $(91.3 \pm 0.49 \%$ inhibition) showing a great potential as a new drug lead, since it was more active than the positive control, miconazole ( $88.7 \pm 2.41 \%$ inhibition). Regarding bacterial inhibition, compounds demonstrated mild activity, but inhibition of compounds $\mathbf{9 ,} \mathbf{1 0}$ and $\mathbf{1 3}$ towards $E$. coli is of interest since it is difficult to find drugs selectively active against Gram (-) bacteria. Most of the compounds were very active in the acetylcholinesterase inhibition assay. Compound 7 was again the most active $(93.2 \pm 4.47 \%)$, being more potent than the control galantamine $(90.3 \pm 0.45 \%)$. The most active gallic acid derivatives, compounds $\mathbf{3}, \mathbf{7}$ and $\mathbf{8}$ have in common, besides gallic acid skeleton, a $\left(\mathrm{CH}_{2}\right)_{2} \mathrm{OH}$ group, which may be one of the structural requirements for $\mathrm{AChE}$ inhibition.
\end{abstract}

Key words: acetylcholinesterase inhibition, antimicrobial activity, indole acid derivatives, 3,4,5-trimethoxybenzoic acid derivatives.

\section{INTRODUCTION}

Natural products offer an unparalleled source of structural diversity and, with little overlap with modified synthetic compounds, are able to provide substances with many biological activities. Gallic acid, an important natural compound found in

Correspondence to: Maria Amélia D. Boaventura

E-mail: boaventuram3@gmail.com

* Contribution to the centenary of the Brazilian Academy of Sciences. several different vegetable sources, has been a building block for different pharmaceutical leads since its skeleton is present in several bioactive natural products. It has been reported to possess anticarcinogenic, antimutagenic, anti-allergic and anti-inflammatory activities (Negi et al. 2005). This acid and its derivative, 3,4,5-trimethoxy benzoic acid (2), showed high antibacterial, antifungal (Bisignano et al. 2000) and antiparkinson activities (Kasture et al. 2009), the latter activity being stronger for $\mathbf{2}$ than for gallic acid. 
Indole derivatives, such as indole-3-acetic acid (plant hormone from auxin class), tryptophan (essential amino-acid), serotonin (neurotransmitter found in all bilateral animals) and melatonin (hormone found in animals, plants and microorganisms) occur widely in natural products, existing in different kinds of plants, animals and marine organisms. The indole core, a major focus of research for generations, is well known as one of the most important scaffolds for drug discovery, being capable of serving as ligand for a diverse array of receptors. Plant growth regulation, antibacterial, antifungal, antihypertensive, antiviral, antimalarial, anti-HIV, anticancer and antioxidant properties have been reported to be associated with the indole nucleus (Zhang et al. 2015).

Amino alcohols are very important and versatile compounds with significant applications in many fields, such as synthetic and medicinal chemistry. Different compounds containing this moiety have been synthesized to use in various diseases. For example, amino alcohols core has the capacity to inhibit aspartic protease enzymes and are widely used as anti-HIV, antimalarial and antileishmaniose agents. The amino alcohol ethambutol is used in the treatment of tuberculosis (Cunico et al. 2011). Whereas amino alcohols inhibit C. albicans and of some filamentous fungi, their amides did not present this effect (Caneschi et al. 2017).

The key target in the management of Alzheimer disease (AD) is the cholinergic hypothesis. According to this hypothesis, inhibition of acetylcholinesterase (AChE), an enzyme that catalyses acetylcholine hydrolysis, increases the levels of acetylcholine in the brain, thus improving cholinergic functions in AD patients. Most of the drugs currently available for treatment of $\mathrm{AD}$ are AChE inhibitors (AChEi), as for example, rivastigmine and galanthamine, which have limited effectiveness and side effects. Rivastigmine was designed from the lead compound physostigmine, a natural AChEi alkaloid, while galanthamine is a natural alkaloid first obtained from Galanthus spp. Several reviews on the newly discovered AChEi obtained from plants, fungi and marine organisms have been published over the last years. The majority of these AChEi belong to the alkaloid group, including indole alkaloids (Murray et al. 2013).

Some serious and life threatening diseases are caused by bacteria and fungal infections. Infections caused by Candida, for example, are responsible for about $80 \%$ of hospital systemic fungal infections, with an overall mortality rate ranging from $40-60 \%$ (Teles et al. 2013). Several classes of chemotherapeutic agents have been introduced in the last decades but the emergence of antibioticresistant bacterial and fungal strains occurred mostly due to the careless use and overconsumption of antibiotics in both human and veterinary medicine (Swamy et al. 2015). Therefore, there is an urgent need for new antimicrobial drugs preferably with new modes of action to potentially avoid crossresistance.

In the design of new drugs, the combination of different pharmacophores in one frame may lead to compounds with interesting pharmacological properties. Also, the co-administration of the moieties, acting by different mechanisms may have synergistic effect, resulting in higher activity than the compounds individually.

The aim of this work was to synthesize hydroxyl amides of 3,4,5-trimethoxybenzoic acid, and 2- and 3-indolecarboxylic acids with six amino alcohols to evaluate their activity as $\mathrm{AChE}$ inhibitors or as antimicrobial and antifungal agents.

\section{MATERIALS AND METHODS}

\section{GENERAL}

Nuclear Magnetic Resonance (NMR) spectra (1D and 2D) were recorded in $\mathrm{CD}_{3} \mathrm{OD}$ and $\mathrm{CDCl}_{3}$, at room temperature, on a Bruker Avance DRX 200 and on a Brucker Avance DRX 400 
spectrometers, from Bruker (Ettlingen, Germany). Electrospray Ionization Mass Spectra (ESI-MS) of compounds was performed using an IT-TOF Apparatus (Shimadzu Corporation, Kyoto, Japan). Methanol was used as solvent. Melting points were determined with a Kofler hot plate apparatus and are uncorrected.

\section{ISOLATION OF ETHYL GALLATE (1)}

Ethyl gallate (1) was isolated from ethanol extract from mesocarp of Caryocar brasiliensis Camb., according to Ascari et al. (2010), with 14\% yield. The grinded mesocarp of $C$. brasiliensis fruits was extracted with ethanol at room temperature for 14 days. The extract $(256.0 \mathrm{~g})$ was subjected to successive column chromatography purification to furnish ethyl gallate, eluted from $\mathrm{CH}_{2} \mathrm{Cl}_{2}$ fractions (1.4 g, 14\% yield), and identified by spectroscopic methods, in comparison with reported values (Ascari et al. 2010). Indolic acid esters were purchased from Sigma-Aldrich (St. Louis, EUA).

SYNTHESIS OF 3,4,5-TRIMETHOXYBENZOIC ACID (2)

Diazomethane was prepared according to methodology described by Hrádková et al. (2013). From $7.0 \mathrm{~g}$ of ethyl gallate, $4.40 \mathrm{~g}$ of pure ethyl 3,4,5-trimethoxy benzoate were obtained (63.5\%), after purification by column chromatography. This product was refluxed with $5 \%$ methanolic $\mathrm{KOH}$ $(16 \mathrm{~mL})$ for $4 \mathrm{~h}$, followed by acidification with $\mathrm{H}_{2} \mathrm{SO}_{4} 40 \%$ (Ozawa et al. 1996). After purification by column chromatography, $2.0 \mathrm{~g}(75.6 \%)$ of 3,4,5-trimetoxybenzoic (2) was obtained (Figure 1).

\section{GENERAL SYNTHETIC PROCEDURE FOR AMIDES} 3-15

A mixture of triphenylphosphine $(527.0 \mathrm{mg}, 2.0$ mmol) and indole acids (2-indolecarboxylic, 3 -indoleacetic, 3-indolepropionic acid) or 3,4,5-trimethoxybenzoic acid 2 (302.0 mg, 1.0 $\mathrm{mmol})$, in dichloromethane $(10.0 \mathrm{~mL})$ was cooled to $0-5{ }^{\circ} \mathrm{C}$. $\mathrm{N}$-Bromosuccinimide $(410.0 \mathrm{mg}, 2.5$ mmol) was added, the reaction mixture was stirred for $15 \mathrm{~min}$, and then, the amino alcohol (1.0-7.0 mmol) was directly introduced (Bandgar and Bettigeri 2004). The temperature was kept during and after the amine addition at $0-5{ }^{\circ} \mathrm{C}$ and the stirring was continued for $30 \mathrm{~min}$. After removal of solvent, the residue was chromatographed on a silica gel column and eluted with solvents with ascending polarities (hexane/dichloromethane/ ethyl acetate/methanol). The products were finally purified by column chromatography with hexane/ dichloromethane /ethyl acetate or Sephadex LH-20 (chloroform/methanol 6:4). The synthetic route for compounds 3-15 is depicted in Figure 1.
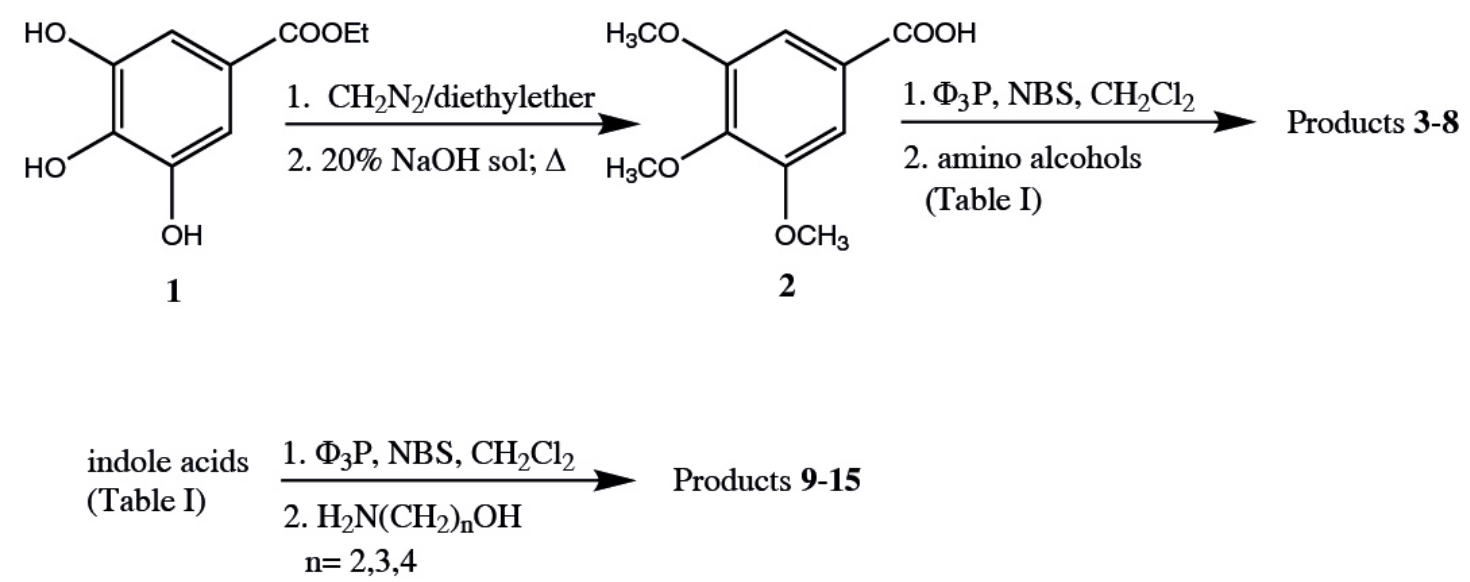

Figure 1 - Synthesis of hydroxyl amides 3-15. 
The structures of all synthesized compounds were confirmed by IR, ${ }^{1} \mathrm{H}-\mathrm{NMR},{ }^{13} \mathrm{C}$-NMR monoand bidimensional, and ESI-MS data. Three of these compounds $(\mathbf{7}, \mathbf{8}$ and $\mathbf{1 5})$ are reported herein for the first time. The structures of all compounds are presented in Figure 2. Yields, ${ }^{1} \mathrm{H},{ }^{13} \mathrm{C}$ NMR and ESI-MS data of all compounds are shown below:

N-(2-HYDROXYETHYL)-3,4,5-TRIMETHOXYBENZAMIDE (3). Yield: $75 \%$. Mp 124-125 ${ }^{\circ} \mathrm{C}$ lit. 118-120 ${ }^{\circ} \mathrm{C}$ (Holerca and Percec 2000). ${ }^{1} \mathrm{H}$ NMR (400 MHz, $\mathrm{CDCl}_{3}$ ), $\delta: 7.01$ (s, H-2, H-6), 6.90 (brs, NH), 3.87 (s, H-9), $\delta 3.86$ (s, H-8), 3.80 (t, J

Acid<smiles>COc1cc(C(=O)O)cc(OC)c1OC</smiles><smiles>COc1cc(C(=O)O)cc(OC)c1OC</smiles><smiles>COc1cc(C(=O)O)cc(OC)c1OC</smiles><smiles>[R]c1[nH]c2ccccc2c1Br</smiles>

Amine alcohol

$\mathrm{H}_{2} \mathrm{~N}\left(\mathrm{CH}_{2}\right)_{\mathrm{n}} \mathrm{OH}$ $\mathrm{n}=2,3,4$

$\mathrm{H}_{2} \mathrm{NC}\left(\mathrm{CH}_{3}\right)_{2} \mathrm{CH}_{2} \mathrm{OH}$
= 4.8 Hz, H-2'), 3.59 (brq, $J=5.2 \mathrm{~Hz}, \mathrm{H}-1$ ') $), 3.07$ (brs, $\mathrm{OH}) ;{ }^{13} \mathrm{C} \mathrm{NMR}\left(100 \mathrm{MHz}, \mathrm{CDCl}_{3}\right), \delta: 168.33$ $(\mathrm{C}=\mathrm{O}), 153.09$ (C-3, C-5), 140.96 (C-4); 129.49 (C-1), 104.47 (C-2, C-6), 62.13 (C-2'), 60.85 (C-9), 56.24 (C-8), 42.95 (C-1'); ESI-MS: m/z 254.1058 $\left([\mathrm{M}-\mathrm{H}]^{-}\right)$(calc. for $\mathrm{C}_{12} \mathrm{H}_{16} \mathrm{NO}_{5}$ 254.1034).

N-(3-HYDROXYPROPYL)-3,4,5-TRIMETHOXYBENZAMIDE (4). Yield: 70\%. Mp 112-115 ${ }^{\circ} \mathrm{C} .{ }^{1} \mathrm{H}$ NMR (400 MHz, $\mathrm{CDCl}_{3}$ ), $\delta: 7.03$ (s, H-2, H-6), 3.87 (s, H-8, H-9), 3.72 (t, $J=5.6$ Hz, H-3'), 3.63-3.58 (m, H-1'), 3.23 (brs, OH), 1.79 (quintet, $\left.J=5.6 \mathrm{~Hz}, \mathrm{H}-2{ }^{\prime}\right) ;{ }^{13} \mathrm{C} \mathrm{NMR}\left(100 \mathrm{MHz}, \mathrm{CDCl}_{3}\right)$,

Product<smiles>COc1cc(C(=O)NCO)cc(OC)c1OC</smiles><smiles>COc1cc(C(=O)NCCO)cc(OC)c1OC</smiles><smiles>[R][R]#N</smiles>

$\mathrm{H}_{2} \mathrm{~N}\left(\mathrm{CH}_{2}\right)_{\mathrm{n}} \mathrm{OH}$ $\mathrm{n}=2,3,4$<smiles>[R]c1[nH]c2ccccc2c1Br</smiles>

9: $\mathrm{R}_{1}=\mathrm{H}, \mathrm{R}_{2}=\mathrm{COOH}, \mathrm{n}=2$

10: $\mathrm{R}_{1}=\mathrm{H}, \mathrm{R}_{2}=\mathrm{COOH}, \mathrm{n}=3$

11: $\mathrm{R}_{1}=\mathrm{H}, \mathrm{R}_{2}=\mathrm{COOH}, \mathrm{n}=4$

12: $\mathrm{R}_{1}=\mathrm{CH}_{2} \mathrm{COOH}, \mathrm{R}_{2}=\mathrm{H}, \mathrm{n}=2$

13: $\mathrm{R}_{1}=\left(\mathrm{CH}_{2}\right)_{2} \mathrm{COOH}, \mathrm{R}_{2}=\mathrm{H}, \mathrm{n}=2$

14: $\mathrm{R}_{1}=\left(\mathrm{CH}_{2}\right)_{2} \mathrm{COOH}, \mathrm{R}_{2}=\mathrm{H}, \mathrm{n}=3$ 15: $\mathrm{R}_{1}=\left(\mathrm{CH}_{2}\right)_{2} \mathrm{COOH}, \mathrm{R}_{2}=\mathrm{H}, \mathrm{n}=4$

Figure 2 - Structure of acids, amino alcohols and hydroxyl amides 3-15. 
$\delta: 168.71(\mathrm{C}=\mathrm{O}), 153.13(\mathrm{C}-3, \mathrm{C}-5), 140.96(\mathrm{C}-$ 4), 129.45 (C-1), 104.41 (C-2, C6), 60.84 (C-9), 59.84 (C-3'), 56.22 (C-8), 37.35 (C-1'), 31.96 (C-2'); ESI-MS: $m / z 268.1187$ ([M-H]') (calc. for $\mathrm{C}_{13} \mathrm{H}_{18} \mathrm{NO}_{5}$ 268.1290).

N-(4-HYDROXYBUTYL)-3,4,5-TRIMETHOXYBENZAMIDE (5). Yield: 72\%. Mp 137-139 ${ }^{\circ} \mathrm{C}$. ${ }^{1} \mathrm{H}$ NMR (400 MHz, $\mathrm{CDCl}_{3}$ ), $\delta: 7.45$ (brs, NH), 7.10 (s, H-2, H-6), 3.89 (s, H-8), 3.86 (s, H-9); 3.66 ( $\mathrm{t}, J=6.0 \mathrm{~Hz}, \mathrm{H}-4$ '), 3.44 (q, $J=6.4 \mathrm{~Hz}, \mathrm{H}-1$ '), 2.87 (brs, $\mathrm{OH}$ ), 1.72 (quintet, $J=6.8 \mathrm{~Hz}, \mathrm{H}-2^{\prime}$ ), 1.66 (quintet, $\left.J=6.4 \mathrm{~Hz}, \mathrm{H}-3^{\prime}\right)$; ${ }^{13} \mathrm{C} \mathrm{NMR}(100$ $\left.\mathrm{MHz}, \mathrm{CDCl}_{3}\right), \delta: 167.04(\mathrm{C}=\mathrm{O}), 152.76(\mathrm{C}-3, \mathrm{C}-5)$, 140.41 (C-2, C-6), 140.32 (C-4) 61.77 (C-4'), 60.59 (C-9), 56.05 (C-8), 39.75 (C-1'), 29.76 (C3'), 25.99 (C-2'); ESI-MS: $m / z$ 284.1488 ([M+H] $\left.]^{+}\right)$ (calc. for $\mathrm{C}_{14} \mathrm{H}_{22} \mathrm{NO}_{5}$ 284.1493).

N-(2-HYDROXY-1,1-DIMETHYLETHYL)3,4,5-TRIMETHOXY-BENZAMIDE (6). Yield: 57\%. Mp 103-104 ${ }^{\circ} \mathrm{C}$ lit. $101-103{ }^{\circ} \mathrm{C}$ (Okuma et al. 2013). ${ }^{1} \mathrm{H}$ NMR (200 MHz, $\left.\mathrm{CDCl}_{3}\right), \delta: 6.95$ (s, H-2, H-6), 3.88 (s, H-8), 3.86 (s, H-9), 3.66 (s, H-2'), 1.41 (s, H-1"); ${ }^{13} \mathrm{C}$ NMR (50 MHz, $\mathrm{CDCl}_{3}$ ),

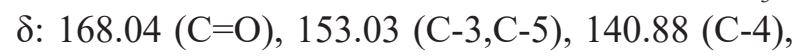
104.39 (C-2, C-6), 70.57 (C-2'), 60.83 (C-9) 56.27 (C-8, C-1'), 24.39 (C-1"); ESI-MS: $m / z$ 284.1285 $\left([\mathrm{M}+\mathrm{H}]^{+}\right)\left(\right.$calc. for $\mathrm{C}_{14} \mathrm{H}_{22} \mathrm{NO}_{5}$ 284.1493).

$N$-(3,4,5-TRIMETHOXYBENZOYL)-4-(2HYDROXYETHYL)-PIPERIDINE (7). Yield: 69.5\%. Mp 83-85 ${ }^{\circ} \mathrm{C} .{ }^{1} \mathrm{H}$ NMR (200 MHz, $\mathrm{CDCl}_{3}$ ),

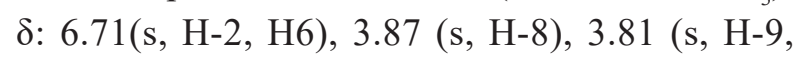
H-2”'), 3.87 (s, H-10), 3.38/3.88 (H-1', H-2', ax/ eq), 2.70 (H-3', H4'), 1.25 (H-5'), 1.80 (H-1'”); ${ }^{13} \mathrm{C}$ NMR (100 MHz, $\left.\mathrm{CD}_{3} \mathrm{OD}\right), \delta: 172.01(\mathrm{C}=\mathrm{O}), 154.78$ (C-3, C-5), 140.57 (C-4), 132.01 (C-1), 105.56 (C2, C-6), 56.89 (C-8, C-10), 61.21 (C-9), 40.21 (C1'), 48.38 (C-2'), 31.57 (C-3', C-4'), 30.61 (C-5'), 35.78 (C-1”), 61.21 (C-2”); ESI-MS: m/z 324.1821 $\left([\mathrm{M}+1]^{+}\right)$(calc. for $\left.\mathrm{C}_{17} \mathrm{H}_{26} \mathrm{NO}_{5} 324.1806\right)$.

$N$-(3,4,5-TRIMETHOXYBENZOYL)-4-(2HYDROXYETHYL)-PIPERAZINE (8). Yield: $75 \%$. Mp 92-94 ${ }^{\circ} \mathrm{C} .{ }^{1} \mathrm{H}$ NMR (400 MHz, $\left.\mathrm{CD}_{3} \mathrm{OD}\right)$, $\delta: 6.70$ (s, H-2, H6), 3.86 (s, H-8), 3.79 (s, H-9), 3.69 (t, $J=6.4 \mathrm{~Hz}, \mathrm{H}-1$ ', H-1'), 3.57 (brs, H-2'), 2.57 (t, $J=8.6 \mathrm{~Hz}, \mathrm{H}-3$ ', H-4', H-2"'); ${ }^{13} \mathrm{C}$ NMR (100 MHz, CD $\mathrm{OD}), \delta: 171.94(\mathrm{C}=\mathrm{O}), 154.58(\mathrm{C}-$ 3, C-5), 140.30 (C-4), 132.04 (C-1), 105.44 (C-2, C-6), 58.60 (C-8), 61.11 (C-9), 42.86 (C-1'), 48.28 (C-2'), 53.86 (C-3', C-4'), 59.29 (C-1”), 60.85 (C2"); ESI-MS: $m / z 325.1716\left([\mathrm{M}+\mathrm{H}]^{+}\right)$(calc. for $\left.\mathrm{C}_{16} \mathrm{H}_{25} \mathrm{~N}_{2} \mathrm{O}_{5} 325.1758\right)$.

N-(2-HYDROXYETHYL)-1H-INDOLE-2CARBOXAMIDE (9). Yield: $74 \%$. Mp 170-172 ${ }^{\circ} \mathrm{C}$ lit. $165-167^{\circ} \mathrm{C}$ (Vlasova et al. 1992). ${ }^{1} \mathrm{H}$ NMR (400 $\mathrm{MHz}, \mathrm{CD}_{3} \mathrm{OD}$ ), $\delta: 7.63$ (d, $\left.J=8.0 \mathrm{~Hz}, \mathrm{H}-4\right), 7.48$ (d, $J=8.0 \mathrm{~Hz}, \mathrm{H}-7), 7.23$ (brt, $J=7.8 \mathrm{~Hz}, \mathrm{H}-6$ ), 7.06-7.10 (m, H-3, H-5), 3.76 (t, $J=5.8$ Hz, H-2'), $3.56\left(\mathrm{t}, J=5.8 \mathrm{~Hz}, \mathrm{H}-1^{\prime}\right)$; ${ }^{13} \mathrm{C}$ NMR $(100 \mathrm{MHz}$, $\left.\mathrm{CD}_{3} \mathrm{OD}\right), \delta: 164.53(\mathrm{C}=\mathrm{O}), 138.34$ (C-7a), 132.23 (C-2), 129.04 (C-3a), 125.06 (C6), 122.76 (C-4), 121.18 (C-5), 113.06 (C-7), 104.50 (C--3), 61.18 (C2'), 43.17 (C-1'); ESI-MS: m/z 203.0794 ([M$\mathrm{H}]^{-}$) (calc. for $\mathrm{C}_{11} \mathrm{H}_{11} \mathrm{~N}_{2} \mathrm{O}_{2}$ 203.0826).

N-(3-HYDROXYPROPYL)-1H-INDOLE-2CARBOXAMIDE (10). Yield: 79\%. Mp 148-151 ${ }^{\circ} \mathrm{C}$ lit. $155-156{ }^{\circ} \mathrm{C}$ (Chacun-Lefèvre et al. 2002). ${ }^{1} \mathrm{H}$ NMR (400 MHz, CD 3 OD), $\delta: 7.62$ (d, $J=8.0 \mathrm{~Hz}$, H-4), 7.47 (d, $J=8.4 \mathrm{~Hz}, \mathrm{H}-7$ ), 7.23 (brt, $J=8.0$ Hz, H-6), 7.10-7.07 (m, H-3, H-5), 3.70 (t, $J=6.0$ $\mathrm{Hz}, \mathrm{H} 3$ '), 3.53 (t, $J=7.0 \mathrm{~Hz}, \mathrm{H}-1$ '), 1.88 (quintet, $J$ $=6.8 \mathrm{~Hz}, \mathrm{H}-2$ ') ${ }^{13} \mathrm{C}$ NMR (100 MHz, CD $\left.\mathrm{OD}\right), \delta$ : $164.42(\mathrm{C}=\mathrm{O}), 138.32$ (C-7a), 132.30 (C-2), 129.06 (C-3a), 125.03 (C-6), 122.73 (C-4), 121.18 (C-5), 113.06 (C-7), 104.25 (C-3), 60.63 (C-3'), 37.69 (C1'), 33.47 (C-2'); ESI-MS: $m / z 217.0953$ ([M-H]') (calc. for $\mathrm{C}_{12} \mathrm{H}_{13} \mathrm{~N}_{2} \mathrm{O}_{2}$ 217.0983).

N-(4-HYDROXYBUTYL)-1H-INDOLE-2CARBOXAMIDE (11). Yield: 74\%. Mp 138-141 ${ }^{\circ} \mathrm{C}$ lit. 141-143 ${ }^{\circ} \mathrm{C}$ (Wang et al. 2006). ${ }^{1} \mathrm{H}$ NMR (400 MHz, CD 3 OD) $\delta: 7.62(\mathrm{~d}, J=8.0 \mathrm{~Hz}, \mathrm{H}-4)$, 7.47 (d, $J=8.4 \mathrm{~Hz}, \mathrm{H}-7), 7.23$ (brt, $J=8.0 \mathrm{~Hz}, \mathrm{H}-6$ ), 7.10-7.06 (m, H-3,H-5), 3.64 (t, $J=6.2$ Hz, H-4'), 3.45 (t, $\left.J=6.8 \mathrm{~Hz}, \mathrm{H}-1^{\prime}\right), 1.77-1.64$ (m, H2', H-3'); ${ }^{13} \mathrm{C}$ NMR (100 MHz, CD $\left.\mathrm{OD}\right), \delta: 161.54(\mathrm{C}=\mathrm{O})$, 
135.61 (C-7a), 129.71 (C-2), 126.37 (C-3a), 122.30 (C-6), 120.02 (C-4), 118.47 (C-5), 110.36 (C-7), 101.55 (C-3), 59.97 (C-4'), 37.74 (C-1'), 28.37 (C3'), 24.53 (C2'); ESI-MS: m/z 231.1112 ([M-H]') (calc. for $\mathrm{C}_{13} \mathrm{H}_{15} \mathrm{~N}_{2} \mathrm{O}_{2}$ 231.1139).

N-(2-HYDROXYETHYL)-1H-INDOLE-3ACETAMIDE (12). Yield: $82 \%$. Viscous oil. ${ }^{1} \mathrm{H}$ NMR (400 MHz, CD OD), $\delta: 7.53(\mathrm{~d}, J=8.0 \mathrm{~Hz}$, H-4), 7.35 (d, $J=8.0 \mathrm{~Hz}, \mathrm{H}-7), 7.15$ (s, H-2), 7.09 (td, $J=8.0,6.8$, and $1.2 \mathrm{~Hz} ; \mathrm{H}-6) ; 7.01$ (td, $J=9.2$, 8.0, and $1.2 \mathrm{~Hz}, \mathrm{H}-5), 3.65(\mathrm{~s}, \mathrm{H}-\alpha), 3.53$ (t, $J=5.8$ $\mathrm{Hz}, \mathrm{H}-2$ ') 3.27 (t, $J=5.8 \mathrm{~Hz}, \mathrm{H}-1$ ') ${ }^{13} \mathrm{C}$ NMR (100 $\left.\mathrm{MHz}, \mathrm{CD}_{3} \mathrm{OD}\right), \delta: 175.31(\mathrm{C}=\mathrm{O}), 138.13(\mathrm{C}-7 \mathrm{a})$, 128.53 (C-3a), 125.11 (C-2), 122.64 (C-6), 120.05 (C-5), 119.40 (C-4), 112.48 (C-7), 109.30 (C-3), 61.57 (C-2'), 43.02 (C-1'), 33.98 (C- $\alpha$ ); ESI-MS: $m / z 219.1081\left([\mathrm{M}+\mathrm{H}]^{+}\right)$(calc. for $\mathrm{C}_{12} \mathrm{H}_{15} \mathrm{~N}_{2} \mathrm{O}_{2}$ 219.1128).

N-(2-HYDROXYETHYL)-1H-INDOLE-3PROPANAMIDE (13). Yield: 76\%. Mp 123-125 ${ }^{\circ} \mathrm{C} .{ }^{1} \mathrm{H}$ NMR (200 MHz, CD $\left.\mathrm{OD}\right), \delta: 7.50$ (d, $J=$ $7.4 \mathrm{~Hz}, \mathrm{H}-4), 7.30$ (d, $J=7.6 \mathrm{~Hz}, \mathrm{H}-7$ ), 7.08-6.92 (m, H-2, H-5, H-6), 3.47 (t, J=5.8 Hz, H-2'), 3.27 (t, $J=5.6 \mathrm{~Hz}, \mathrm{H}-1^{\prime}$ ), 3.02 (t, $\left.J=7.6 \mathrm{~Hz}, \mathrm{H}-\beta\right), 2.56$ (t, $J=7.6 \mathrm{~Hz}, \mathrm{H}-\alpha) ;{ }^{13} \mathrm{C}$ NMR (50 MHz, CD $\left.\mathrm{OD}\right)$, $\delta: 176.21(\mathrm{C}=\mathrm{O}), 138.00(\mathrm{C}-7 \mathrm{a}), 128.41(\mathrm{C}-3 \mathrm{a})$, 122.85 (C-2), 122.18 (C-6), 119.41 (C-5), 119.19 (C-4), 114.96 (C-3), 112.07 (C-7), 61.47 (C-2'), 42.80 (C-1'), 38.18 (C- $\alpha$ ), 22.49 (C- $\beta$ ); ESI-MS: $m / z 231.1093\left([\mathrm{M}-\mathrm{H}]^{-}\right)$(calc. for $\mathrm{C}_{13} \mathrm{H}_{15} \mathrm{~N}_{2} \mathrm{O}_{2}$ 231.1138).

N-(3-HYDROXYPROPYL)-1H-INDOLE-3PROPANAMIDE (14). Yield: 79\%. Viscous oil. ${ }^{1} \mathrm{H}$ NMR (400 MHz, CD $\mathrm{OD}), \delta: 7.54(\mathrm{~d}, J=8.0 \mathrm{~Hz}$, H-4), 7.31 (d, $J=8.0 \mathrm{~Hz}, \mathrm{H}-7), 7.06$ (t, $J=7.2 \mathrm{~Hz}$, H6), 7.00-6.97 (m, H-2, H-5), 4.56 (brs, OH), 3.43 (t, $J=6.2 \mathrm{~Hz}, \mathrm{H}-3^{\prime}$ ), 3.19 (t, $\left.J=6.8 \mathrm{~Hz}, \mathrm{H}-\beta\right), 3.04$ (t, $J=7.6 \mathrm{~Hz}, \mathrm{H}-\alpha), 2.52$ (t, $J=7.4 \mathrm{~Hz}, \mathrm{H}-1^{\prime}$ ), 1.58 (quintet, $\left.J=6.6 \mathrm{~Hz}, \mathrm{H}-2^{\prime}\right) ;{ }^{13} \mathrm{C}$ NMR $(100 \mathrm{MHz}$, $\left.\mathrm{CD}_{3} \mathrm{OD}\right), \delta: 176.17(\mathrm{C}=\mathrm{O}), 138.14(\mathrm{C}-7 \mathrm{a}) 128.54$ (C-3a), 123.07 (C-2) 122.33 (C-6), 119.58 (C-5), 119.36 (C-4), 115.01 (C-3) 112.23 (C-7), 60.28 (C-
3'), 38.35 (C-1'), 37.29 (C- $\alpha$ ), 33.11 (C-2'), 22.70 (C- $\beta$ ); ESI-MS: $m / z 245.1305$ ([M- H] $]^{-}$) (calc. for $\mathrm{C}_{14} \mathrm{H}_{17} \mathrm{~N}_{2} \mathrm{O}_{2}$ 245.1296).

N-(4-HYDROXYBUTYL)-1H-INDOLE-3PROPANAMIDE (15). Yield: 68\%. Mp 127-130 ${ }^{\circ} \mathrm{C} .{ }^{1} \mathrm{H}$ NMR (400 MHz, CD OD), $\delta: 7.54$ (d, $J=8.0$ $\mathrm{Hz}, \mathrm{H}-4), 7.31$ (d, $J=8.0 \mathrm{~Hz}, \mathrm{H}-7), 7.06$ (brt, $J=7.2$ Hz, H-6), 7.01-6.97 (m, H-2, H-5), 4.56 (brs, OH); 3.49 (t, $J=5.8 \mathrm{~Hz}, \mathrm{H}-4$ ') 3.14 (t, $J=6.4 \mathrm{~Hz}, \mathrm{H}-1^{\prime}$ ), $3.11(\mathrm{t}, J=7.6 \mathrm{~Hz}, \mathrm{H}-\beta), 2.55$ (t, $J=7,6 \mathrm{~Hz}, \mathrm{H}-\alpha)$, 1.45-1.38 (m, H-2', H-3'); ${ }^{13} \mathrm{C}$ NMR (100 MHz, $\left.\mathrm{CD}_{3} \mathrm{OD}\right), \delta: 175.94(\mathrm{C}=\mathrm{O}), 138.14(\mathrm{C}-7 \mathrm{a}), 128.57$ (C-3a), 123.07 (C-2), 122.31 (C-6), 119.56 (C-5), 119.34 (C-4), 115.04 (C-3), 112.21 (C-7), 62.53 (C-4'), 40.16 (C-1'), 38.40 (C- $\alpha$ ), 30.76 (C-3'), 26.78 (C-2'), 22.73 (C- $\beta$ ). ESI-MS: $m / z 261.1598$ $\left([\mathrm{M}+\mathrm{H}]^{+}\right)$(calc. for $\mathrm{C}_{15} \mathrm{H}_{21} \mathrm{~N}_{2} \mathrm{O}_{2}$ 261.1598).

\section{EVALUATION OF THE ANTIMICROBIAL ACTIVITY}

For the evaluation of antimicrobial activity, Gram (+) Staphylococcus aureus (ATCC 29212) and Bacilus cereus (ATCC 11778), Gram (-) Pseudomonas aeruginosa (ATCC 27853) and Escherichia coli (ATCC 25922) and yeast Candida albicans (ATCC 18804) were used. The assays were conducted in microplates, in DMSO, and microorganisms were inoculated as suspensions, according to methodology described by Bracarense and Takahashi (2014). The results are presented on Table I.

\section{EVALUATION OF ACETYLCHOLINESTERASE INHIBITORY ACTIVITY}

The assay was made in microplates, based on Ellmann's methodology (Teles et al. 2013). The results are presented on Table II.

\section{RESULTS AND DISCUSSION}

The hydroxyl amides 3-15 were obtained by modified methodology described by Bandgar and Bettigeri (2014). Gallic acid was isolated as ethyl 
TABLE I

Antifungal and antibacterial activity data of hydroxyl amides 3-15, at $250 \mathrm{mg} / \mathrm{mL}$.

\begin{tabular}{cccccc}
\hline Compound & C. albicans & E. coli & P. aeruginosa & S. aureus & B. cereus \\
\hline 3 & $20.3 \pm 2.42$ & $19.7 \pm 2.68$ & $17.3 \pm 1.19$ & $25.6 \pm 0.31$ & 0.0 \\
4 & $20.7 \pm 2.45$ & $24.4 \pm 3.80$ & $16.4 \pm 1.68$ & $23.1 \pm 0.86$ & 0.0 \\
5 & $23.9 \pm 0.48$ & $10.4 \pm 1.90$ & $6.9 \pm 1.89$ & $13.8 \pm 5.84$ & $9.7 \pm 0.59$ \\
6 & $25.3 \pm 0.56$ & $24.2 \pm 1.42$ & $27.7 \pm 3.61$ & $24.4 \pm 2.26$ & $19.8 \pm 0.09$ \\
7 & $91.3 \pm 0.49$ & $22.1 \pm 0.35$ & $17.8 \pm 1.32$ & $26.3 \pm 1.01$ & $18.1 \pm 0.81$ \\
8 & $29.7 \pm 1.20$ & $17.1 \pm 3.19$ & $8.3 \pm 0.79$ & $15.1 \pm 0.88$ & $12.1 \pm 0.89$ \\
9 & $61.0 \pm 2.30$ & $44.0 \pm 1.55$ & $35.6 \pm 2.20$ & $40.3 \pm 0.66$ & $18.1 \pm 4.05$ \\
10 & $63.0 \pm 2.18$ & $49.8 \pm 1.03$ & $31.0 \pm 1.69$ & $36.2 \pm 1.63$ & $26.9 \pm 0.72$ \\
11 & $31.3 \pm 2.95$ & $39.2 \pm 3.89$ & $25.6 \pm 1.39$ & $38.0 \pm 1.09$ & $18.1 \pm 5.04$ \\
12 & $21.7 \pm 1.15$ & $35.4 \pm 2.66$ & $19.9 \pm 2.76$ & $43.0 \pm 0.62$ & $21.7 \pm 0.90$ \\
13 & $21.9 \pm 3.33$ & $47.0 \pm 3.11$ & $34.1 \pm 2.25$ & $47.6 \pm 1.79$ & $23.3 \pm 1.44$ \\
14 & $24.5 \pm 1.27$ & $33.9 \pm 2.81$ & $25.8 \pm 1.68$ & $33.8 \pm 1.22$ & $12.5 \pm 1.71$ \\
15 & $56.6 \pm 2.84$ & $31.9 \pm 2.07$ & $18.7 \pm 0.44$ & $35.6 \pm 0.39$ & $22.7 \pm 2.34$ \\
Miconazole & $88.7 \pm 2.41$ & - & - & - & - \\
Ampiciline & - & $96.3 \pm 0.43$ & $96.5 \pm 0.40$ & $97.9 \pm 0.12$ & $96.8 \pm 0.27$ \\
\hline
\end{tabular}

TABLE II

Inhibition (\%) of acetylcholinesterase by hydroxyl amides 3-15.

\begin{tabular}{cc}
\hline Sample & \% Inhibition $($ mean $\pm \mathrm{sd})$ \\
\hline $\mathbf{3}$ & $86.7 \pm 1.55$ \\
$\mathbf{5}$ & $80.8 \pm 1.94$ \\
$\mathbf{6}$ & $80.8 \pm 1.94$ \\
$\mathbf{7}$ & $87.5 \pm 0.80$ \\
$\mathbf{8}$ & $93.2 \pm 4.47$ \\
$\mathbf{9}$ & $83.6 \pm 5.94$ \\
$\mathbf{1 0}$ & $37.9 \pm 4.25$ \\
$\mathbf{1 1}$ & $20.5 \pm 3.59$ \\
$\mathbf{1 2}$ & $56.5 \pm 3.83$ \\
$\mathbf{1 3}$ & $67.5 \pm 2.90$ \\
$\mathbf{1 4}$ & $37.6 \pm 3.94$ \\
$\mathbf{1 5}$ & $59.3 \pm 4.82$ \\
Galantamine & $71.4 \pm 5.41$ \\
Eserine & $90.3 \pm 0.45$ \\
\hline
\end{tabular}

gallate from ethanol extract from mesocarp of $C$. brasiliense Camb, with very good yield, 14\%, (Ascari et al. 2010) and the methyl ether derivatives were obtained by reaction with diazomethane followed by ester hydrolysis.

\section{ANTIMICROBIAL ACTIVITY}

The percent inhibition data of hydroxyl amides 3-15 against $C$. albicans, E. coli, $P$. aeruginosa, $S$. aureus, and B. cereus are given in Table I, using a concentration of $250 \mathrm{mg} / \mathrm{mL}$. From Table I, it is observed that indole derivatives were very active against the yeast $C$. albicans. This microorganism, nevertheless opportunistic, can cause life-threatening infections under suitable conditions. Among the indole derivatives assayed, compound 10 was the most active $(63 \%)$, followed by compound $9(61 \%)$ and compound $15(56.6 \%)$, showing that these derivatives were able to inhibit C. albicans growth regardless the size of the side chain. It is very noteworthy to point that gallic acid derivatives activity against $C$. albicans was very modest, except for compound 7 , which presented an outstanding activity even higher than miconazole, the clinically used drug utilized as a control in the experiments. This activity resulted from the introduction of a side chain containing the amide nitrogen bearing a six ring unit. The presence of a second nitrogen atom in this ring decreases the 
activity drastically. Since this compound is reported for the first time in the literature, new antimicrobial leads detected in our work deserves further studies as potential new selective antibiotics to be used in therapeutics.

Compounds 9 (44\%), 10 (49\%) and 15 (47\%) were active against E. coli, a Gram (-) bacteria. These results are of great interest since Gram (-) bacteria are becoming increasingly resistant to the known antibiotics and development of new drugs is an urgent need. E. coli is a concern, not only because it causes threatening diseases such as pneumonia and meningitis, but also because of opportunistic bloodstream infections associated to wounds and surgical infections. The consistence of activity of the indole derivatives assayed against $E$. coli indicates these compounds as promising drug leads to the development of new antibiotics. The most active compounds towards $S$. aureus were the indole derivatives 9 (40.3\%) and 15 (47.6\%), showing that the size of the side chain from the amino alcohol moiety the most important factor for the activity. Also, compound $\mathbf{1 2}$ rises interest, since it was not very active against the other microorganisms assayed, showing therefore, some selectivity against $S$. aureus. Since this bacterial strain is well known to be resistant to clinically used antibiotics, this selectivity can be further exploited in the search for antibiotics active against resistant strains. In general, all compounds displayed weak antibacterial activity against $B$. cereus, a Gram (+) bacterium, and $P$. aeruginosa, a Gram (-) one. Gallic acid derivatives 3-8 were not significant active neither against $S$. aureus nor against the other bacteria assayed. Corroborating to our results, indoleamide derivatives were indeed reported to be highly active against $E$. coli, S. aureus, C. albicans and B. subtilis, when tested by the disc diffusion method (Ölgen et al. 2008). On the other side, contrary to our results, where 3,4,5-trimethoxybenzoic acid was inactive, in the literature it shows high antibacterial and antifungal activities, against $S$. aureus and $C$. albicans (Bisignano et al. 2000). This observed lack of activity could be due to the fact that, according to Caneschi et al. (2017), whereas amino alcohols presented activity against fungi, such as $C$. albicans, amides from these amino alcohols did not show any effect.

\section{ANTIACETYLCHOLINESTERASE ACTIVITY}

The compounds synthesized in this work were also assayed for acetylcholinesterase (AChE) inhibitory activity (Table II). Biological screenings targeting a single activity can, many times, ignore the whole potential of a group of compounds. This is well exemplified by the results obtained for $\mathrm{AChE}$ inhibition. While the indole derivatives showed, in general, more activity against microorganisms than 3,4,5-trimethoxybenzoic acid derivatives, the latter were consistently more active as AChE inhibitors than the former. While the literature cited no significant AChE activity for gallic acid (Orhan et al. 2007), indole alkaloids presented good AChE activity (Murray et al. 2013). Among the indole derivatives able to inhibit $\mathrm{AChE}$, the most active was compound $\mathbf{1 5}$, which bears the longer side chain, from amino alcohol moiety. The higher activity of $\mathbf{1 5}$ may be related to the possibility of forming intramolecular hydrogen bonds leading the compound to assume a spatial volume closer to that of galantamine, a bulk four rings molecule. However, concerning the side chain derived from the amino alcohol moiety, in 3,4,5-trimethoxybenzoic acid derivatives, increase of methylene groups did not improve the activity since compound $\mathbf{3}$, with the smaller chain is more active $(86.7 \%)$ than $5(80.8 \%)$. However, as already pointed, gallic acid derivatives were more active than indole compounds in this assay and this can be a result of different action mechanisms. Again, compound 7 showed the higher activity for AChE inhibition, also more potent than positive controls. The most 
active 3,4,5-trimthoxybenzoic acid derivatives, compounds $\mathbf{3}, \mathbf{7}$ and $\mathbf{8}$, have in common, besides the gallic acid skeleton, a $\left(\mathrm{CH}_{2}\right)_{2} \mathrm{OH}$ group, which may be one of the structural requirements for $\mathrm{AChE}$ inhibition. However, it is not the only requirement for the activity, since compound 9 was inactive.

In conclusion, in a general way, the antimicrobial and antifungal activities of 3,4,5-trimthoxybenzoic acid (2) derivatives were decreased by the introduction of the amino alcohol moieties, compared to acid $\mathbf{2}$ alone. On the other side, these moieties increased the AChE activity of these derivatives. Then this work showed that relatively simple modifications in the structure of natural products derivatives can bring about novel pharmacological potential for known molecules.

\section{ACKNOWLEDGMENTS}

The authors thank Conselho Nacional de Desenvolvimento Científico e Tecnológico (CNPq) for MADB and JAT grants.

\section{REFERENCES}

ASCARI J, TAKAHASHI J AND BOAVENTURA MAD. 2010. Phytochemical and biological investigations of Caryocar brasiliense Camb. B Latinoam Caribe PL 9: 20-28.

BANDGAR BP AND BETTİGERİ SV. 2004. Direct synthesis of $N$-acylalkylenediamines from carboxylic acids under mild conditions. Synth Commun 34: 2917-2924.

BISIGNANO G, SANOGO R, MARINO A, AQUINO R, D`ANGELO V, GERMANO MP, PASQUALE DER AND PIZZA C. 2000. Antimicrobial activity of Mitracarpus scaber extract and isolated constituents. Lett Appl Microbiol 30: 105-108.

BRACARENSE AAP AND TAKAHASHI JA. 2014. Modulation of antimicrobial metabolites production by the fungus Aspergillus parasiticus. Braz J Microbiol 45: 313-321.

CANESCHI CA, ALMEIDA AM, MARTINS FJM, LE HYARIC M, OLIVEIRA MME, MACEDO GC, ALMEIDA MV AND RAPOSO NRB. 2017. In vitro antifungal activity of organic compounds derived from amino alcohols against onychomycosis. Braz J Microbiol 48: 476-482.

CHACUN-LEFÈVRE L, BÉNÉTEAU V, JOSEPH B AND MÉROUR JY. 2002. Ring closure metathesis of indole 2-carboxylic acid allylamide derivatives. Tetrahedron 58: 10181-10188.

CUNICO W, GOMES CRB, FERREIRA MLG, FERREIRA TG, CARDINOT D, SOUZA MVN AND LOURENÇO MCS. 2011. Synthesis and anti-mycobacterial activity of novel amino alcohol derivatives. Eur J Med Chem 46: 974978.

HOLERCA MN AND PERCEC V. 2000. 1H NMR Spectroscopic Investigation of the Mechanism of 2-Substituted-2-Oxazoline Ring Formation and of the Hydrolysis of the Corresponding Oxazolinium Salts. Eur J Org Chem 12: 2257-2263.

HRÁDKOVÁ I, MERKL R, SMIDRKAL J, KYSELKA J AND FÍLIP V. 2013. Antioxidant effect of monoand dihydroxyphenols in sunflower oil with different levels of naturally present tocopherols. Eur J Lipid Sci Technol 115: 747-755.

KASTURE VS, KATTI SA, MAHAJAN D, WAGH R, MOHAN M AND KASTURE SB. 2009. Antioxidant and Antiparkinson Activity of Gallic Acid Derivatives. Pharmacologyonline 1: 385-395.

MURRAY AP, FARAONI MB, CASTRO MJ, ALZA NP AND CAVALLARO V. 2013. Natural AChE Inhibitors from Plants and their Contribution to Alzheimer's Disease Therapy. Curr Neuropharmacol 11: 388-413.

NEGİ AS, DAROKAR MP, CHATTOPADHYAY SK, GARG A, BHATTACHARYA AK, SRIVASTAVA V AND KHANUJA SPS. 2005. Synthesis of a novel plant growth promoter from gallic acid. Bioorg Med Chem Lett 15: 1243-1247.

OKUMA AA, ANTONIO DC, CARAZZA F AND DUARTE LP. 2013. Microwave assisted green synthesis of aryl methoxylated benzamides and 2-oxazolines from biomass. Green Process Synth 2: 51-56.

ÖLGEN S, ALTANLAR N, KARATAYLI E AND BOZDAYI M. 2008. Antimicrobial and Antiviral Screening of Novel Indole Carboxamide and Propanamide Derivatives. Z Naturforsch 63: 189-195.

ORHAN I, KARTAL M, TOSUN F AND SENER B. 2007. Screening of Various Phenolic Acids and Flavonoids Derivatives for their Anticholinesterase Potential. Z Naturforsh 62: 829-832.

OZAWA T, KATAOKA M, MORIKKAWA K AND NEGİSHİ O. 1996. Elucidation of the Partial Structure of Polymeric Thearubigins from Black Tea by Chemical Degradation. Biosci Biotech Biochem 60: 2023-2027.

SWAMYPV,CHANDRASEKHARKBANDKAMBHAMPATI PC. 2015. Synthesis and antibacterial activity of novel substituted azetidinones bearing 3,4,5-trimethoxy phenyl group. Der Pharma Chem 7: 338-345.

TELES AP, ATALIBA GS AND TAKAHASHI JA. 2013. Modulation of Paecilomyces lilacinus antimicrobial metabolite production by co-culturing with Salmonella typhimurium. Nat Prod Res 27: 1598-1601. 
VLASOVA MI, KOGAN NA, LESIOVSKAYA EE AND PASTUSHENKOV LV. 1992. Synthesis and Biological Activity of 1-aryl-2-oxa-5-aza-5R 2-6-oxocyclooctano[6,7-b] Indoles. Pharm Chem J 26: 492-496.

WANG JJ, SHEN YK, HU WP, HSIEH MC, LIN FL, HSU MK AND HSU MH. 2006. Design, Synthesis, and Biological
Evaluation of Pyrrolo[2,1-c][1,4]benzodiazepine and Indole Conjugates as Anticancer Agents. J Med Chem 49: 1442-1449.

ZHANG M-Z, CHEN Q AND YANG G-F. 2015. A review on recent developments of indole-containing antiviral agents. Eur J Med Chem 89: 421-441. 